$5-1-2005$

\title{
Neurobehavioral Functioning and Breastfeeding Behavior in The Newborn
}

Sharon Radzyminski

Cleveland State University, s.radzyminski@csuohio.edu

Follow this and additional works at: https://engagedscholarship.csuohio.edu/nurs_facpub

Part of the Nursing Commons

How does access to this work benefit you? Let us know!

\section{Publisher's Statement}

This is the accepted version of the following article: Radzyminski, S. (2005). Neurobehavioral functioning and breastfeeding behavior in the newborn. Journal of Obstetric, Gynecologic, \& Neonatal Nursing, 34(3), 335-341. doi:10.1177/0884217505276283, which has been published in final form at http://onlinelibrary.wiley.com/doi/10.1177/0884217505276283/full

\section{Recommended Citation}

Radzyminski, Sharon, "Neurobehavioral Functioning and Breastfeeding Behavior in The Newborn" (2005). Nursing Faculty Publications. 40.

https://engagedscholarship.csuohio.edu/nurs_facpub/40

This Article is brought to you for free and open access by the School of Nursing at EngagedScholarship@CSU. It has been accepted for inclusion in Nursing Faculty Publications by an authorized administrator of EngagedScholarship@CSU. For more information, please contact library.es@csuohio.edu. 


\section{Neurobehavioral Functioning and Breastfeeding Behavior in the Newborn Sharon Radzyminski}

Objective: To determine whether central nervous system functioning has an effect on the normal, term infant's ability to breastfeed in the first day following birth.

Design: Breastfeeding behaviors and neurobehaviors were evaluated at birth and at $24 \mathrm{hrs}$ of age in two groups of neonates. One group of neonates was born to mothers who received epidural analgesia during labor, and one group was born to mothers who received no pain medication. Breastfeeding behavior was evaluated using the Preterm Infant Breastfeeding Behavior Scale, and the infant's neurobehaviors were evaluated using the Neurologic and Adaptive Capacity Score.

Participants: Fifty-six breastfeeding mothernewborn dyads. All mothers were healthy multiparae who gave birth vaginally to normal, full-term, healthy newborns.

Main Outcome Measures: Newborns were observed for rooting, latch-on, sucking, swallowing, activity state, and neurobehavior.

Result: Analysis of the data indicated that the higher the infant scored in relation to neurobehavioral functioning, the higher the infant scored on breastfeeding behaviors.

Conclusion: When determining physiologically what is important for the infant to successfully latch on and feed, it appears that an intact and functioning central nervous system may be one of the crucial elements. JOGNN, 34, 335-341; 2005. D0I: 10.1177/ 0884217505276283

Keywords: Breastfeeding-CNS functioningInfant feeding-Neurobehaviors
Breastfeeding has been shown to improve infant health, and breast milk has been characterized as the ideal first food for the infant. For these reasons, many women choose to breastfeed. However, there are many who encounter difficulties with the initiation of lactation, and it is estimated that half of aU American mothers who begin breastfeeding wilJ stop within the first 3 weeks (Lawrence, 1994).

The reasons why an infant would exhibit behaviors that are inconsistent with feeding are not fulJy understood. Researchers in the field of lactation have suggested that separation of the mother and infant at birth and labor analgesia (Righard \& Alade, 1990), maternal support systems (Krishna \& Plichta, 1998), and the use of pacifiers, formula, and glucose water in the 1st days after birth (Hill, Hummenick, Brennan, \& Wooley, 1997) may in some way negatively impact the infant's ability to establish good breastfeeding behaviors.

Breastfeeding behaviors crucial to the establishment of lactation include latch-on, or the infant's ability to grasp breast tissue and retain it in the mouth; sucking, or the ability to compress the areolar tissue and express the milk from the ducts; and the coordination of sucking with swallowing and breathing. In addition, the infant needs to be in an awake, alert state. Therefore, it is possible that any factor that inhibits the infant from successfully accomplishing any of these behaviors could potentiaUy create the breastfeeding problems identified by mothers and health care professionals.

Physiologically an intact and functioning central nervous system may be one of the crucial elements for an infant to successfuUy latch on and feed. Cranial nerve function and maturation and reflexes 
manifest themselves in a predictable pattern of behavior in newborn infants that can be used for assessment purposes. These behaviors are commonly called neurobehaviors. Thus, it is reasonable to postulate that whatever affects neurobehavioral responses in the newborn has the potential to affect breastfeeding behaviors. To test this premise, infant neurobehavior was compared to breastfeeding behavior at $1 \mathrm{hr}$ and $24 \mathrm{hrs}$ of life. This study was done in conjunction with a larger study that tested the effect of epidural analgesia on newborn breastfeeding behaviors (Radzyminski, 2003).

\section{Does central nervous system functioning have}

\section{an effect on the normal, term newborn's ability to breastfeed in the 1st days followin birth?}

\section{Setting and Study Methods}

The study was conducted at a large intercity tertiary medical center and its community-based affiliate hospital. Both hospitals followed the same policies and procedures. Standard maternal-infant care included rooming-in and breastfeeding on demand. The study was approved by the IRB at both the tertiary medical center and its communitybased affiliate and Case Western Reserve University.

Selection criteria required the mothers in this study to be at least 18 years of age, have a normal prenatal course, be multiparous, receive no medication during labor and delivery except the standard epidural infusion, have normal, uncomplicated vaginal deliveries, and require no more than standard routine care postpartum. The infants needed to be normal and term with Apgar scores of at least 8 at 1 and $5 \mathrm{~min}$ and have no evidence of in utero or birth asphyxia. AU newborns had to be healthy enough to room-in with their mothers after birth and require no more than routine newborn care.

To remain in the study, all mothers and newborns needed to be discharged within 48 hrs of delivery without complications. In addition, newborns needed to be exclusively breastfeeding at the time of discharge. All study participants met the criteria and were able to complete the study.

One hundred fifty mothers were approached in labor and delivery by their labor and delivery nurse to participate in the study. Recruitment took place 7 days a week. Patients who met the inclusion criteria on admission to labor and delivery were approached to be in the study using a random table of numbers. Participants receiving an even number were included in the study (total of 75 patients), and participants who received an odd number were not (total of 75 patients). Only one patient declined to be in the study. Of the 75 mother-infant dyads who remained in the study, 17 were eliminated from data analysis for failure of the labor and delivery nurse to follow the protocol of placing the newborn immediately on the mother's chest postdelivery. One mother-infant dyad was eliminated because the newborn was intubated postdelivery for meconium-stained fluid, and one was eliminated for shoulder dystocia. Twenty-eight of the mothers delivered by natural childbirth, and 28 delivered under epidural analgesia. The mothers who delivered under epidural analgesia all received the standard infusion of 0.044\% bupivacaine (Marcaine, Sanofi Winthrop Pharmaceuticals, Morrisville, PA) $+0.000125 \%$ fentanyl (Sublirnaze, Abbott Laboratories, Chicago) + 1:800,000 epinephrine at $14 \mathrm{cc} / \mathrm{hr}$ during labor as set by the Department of OB Anesthesia at the study site. Because the amount of time the mother received the infusion varied, cord blood was obtained from all the mothers who received epidural analgesia at delivery for drug concentration analysis. Two variables were examined: length of time the mother received the epidural infusion and the amount of drug present in the cord blood at birth. Variables describing the sample are included in Tables 1 and 2 .

Immediately after delivery, the newborn was placed on the mother's chest and covered by a warmed blanket. The newborn remained skin-to-skin with his or her mother for the 1st hour of life. Apgar scores, vital signs, and assessments were done with the newborn on the mother's chest. AU other assessments such as weights, eye care, vitamin $\mathrm{K}$, and physical exams were delayed until after the 1st hour of skin-to-skin care. During this period, the newborns' initial breastfeeding behaviors were recorded using the Preterm Infant Breastfeeding Behavior Scale (PIBBS). This scale was developed through several procedures and field tested with both full-term and preterm newborns (Nygvist, Rubertson, Ewaksm, \& Sjoden, 1996). Breastfeeding behaviors were selected by observing newborns breastfeed and then interviewing the mothers afterward. Revisions were made following feedback, and content validity was determined by a panel of three breastfeeding experts that included a dietician and two midwives (Nygvist et al., 1996). The PIBBS includes measurements of rooting, sucking, swallowing, the actual number of sucks and sucking bursts, the infant activity state, the mother's awareness of letdown, problems noted by the mother, and any environmental influences. These assessments were indicated as "Behaviors" on the PIBBS.

For the purpose of data collection, each behavior was given a numerical score (for example, the first behavior is "rooting"; no rooting was given a score of 0 , showed some rooting a score of 1 , and showed obvious rooting a score of 2). Full permission was obtained from the author 
TABLE 1

Measures of Central Tendency for Variables Describing the Sample (n = 28 unmedicated; 28 medicated)

\begin{tabular}{|c|c|c|c|c|c|c|}
\hline \multirow[b]{2}{*}{ Variable } & \multicolumn{3}{|c|}{ Unmedicated } & \multicolumn{3}{|c|}{ Medicated } \\
\hline & Range & Mean & $S D$ & Range & Mean & $S D$ \\
\hline Maternal age in years & $19-39$ & 27.5 & 5.28 & $24-43$ & 31 & 4.7 \\
\hline Gravida & $2-8$ & 3 & 1.39 & $2-7$ & 3.5 & 1.45 \\
\hline Parity & $1-6$ & 1.78 & 1.26 & $1-4$ & 1.67 & 0.86 \\
\hline Length of labor (in hours) Stage I & $0.50-16$ & 5.21 & 3.4 & $1-12$ & 6.48 & 4.35 \\
\hline Length of labor (minutes) Stage II & $2-66$ & 15.5 & 5.1 & $3-120$ & 19.6 & 21.2 \\
\hline Newborn birth weight & $2,646-4,120$ & 3,396 & 381 & $2,610-4,943$ & 3,483 & 470 \\
\hline $\begin{array}{l}\text { Newborn feeding (number of feedings } \\
\text { in first } 24 \mathrm{hrs} \text { ) }\end{array}$ & $1-7$ & 4.1 & 2.9 & $3-8$ & 5.4 & 2.6 \\
\hline
\end{tabular}

(Nyquist, personal communication, May 1999) to adapt the tool using the scoring system. Data were collected during the breastfeeding session by the principal investigator who was blind to the study group assignment. There was no time limit placed on how long the newborn could breastfeed. The breastfeeding session was considered ended when the newborn fell asleep or showed no further interest in feeding.

At $1 \mathrm{hr}$ of age, neurobehaviors were measured in the newborn using the Neurologic and Adaptive Capacity Score (NACS) (Amid-Tison, Barrier, \& Shnider, 1982). Prior to 1982, the Early Neonatal Neurobehavioral Scale (ENNS) was considered the gold standard by which to evaluate drug effect on newborns (Scanlon, Brown, Weiss, \& Alper, 1974). Predictive validity was established (Hodgkinson, Bhatt, \& Wang, 1978) on the basis that the ENNS was able to identify narcotic effect on 920 normal, full-term newborns and correctly identified newborns born under epidural analgesia. The NACS and ENNS were compared in a study in which the same newborns were tested and then scored by both instruments (AmielTison et al., 1982). The interobserver reliability was reported at $92.8 \%$ for the NACS and $88 \%$ for the ENNS. The NACS was reported to have concurrent validity with the ENNS $92 \%$ of the time. It was selected for use in this study because of the concurrent validity with the ENNS but was less time consuming and did not contain the repeated pinpricks required by the ENNS.

The NACS included evaluation of response and habituation to light and sound, passive tone, active tone, primary reflexes, and a general assessment. Permission to use the NACS was obtained prior to the onset of the study. The newborns then received normal nursery care and were returned to their mothers. The newborns roomed in with their mothers and were breastfed on demand. At 24 hrs of age, the newborns were again evaluated with the PIBBS and NACS in the exact same manner they were at
TABLE 2

Percentage of Occurrence for Nominal Variables Describing the Sample ( $n=28$ medicated; 28 unmedicated)

\begin{tabular}{|lcc|}
\hline Variable & Umnedicated & Medicated \\
\hline $\begin{array}{l}\text { Previous breastfeeding } \\
\text { expenence }\end{array}$ & $79 \%$ & $82 \%$ \\
$\begin{array}{l}\text { Infant sex (male) } \\
\begin{array}{l}\text { Use of pitocin for labor } \\
\text { augmentation }\end{array}\end{array}$ & $39 \%$ & $68 \%$ \\
\hline
\end{tabular}

$1 \mathrm{hr}$ of age. The newborns were weighed prefeeding and postfeeding at the $24 \mathrm{hrs}$ of age data collection session. All the newborns were exclusively breastfed during the course of the study.

At the conclusion of the study, the medical records of the mothers and newborns were reviewed by the primary investigator, and information related to the labor \& delivery experience, breastfeeding data, and newborn assessments were recorded.

Data were obtained from 56 mother-infant dyads during 112 breastfeeding sessions and 112 neurological examinations of the newborns. All data were obtained by the principal investigator who stayed with the motherinfant dyads during the data collection sessions and recorded and timed all observed behaviors. The principal investigator was blind to whether the mother received an epidural or not. The data were then coded and examined graphically in box plots and within-group histograms to determine whether the distribution of variables was symmetric and free of gross outliers and the spread of data across the groups was fairly constant. Measures of central tendency were calculated, and multiple regression was used to determine the degree of the relationship between the NACS and the breastfeeding behaviors on the PIBBS. 
TABLE 3

$\boldsymbol{R}_{\text {eg }}$ ression Analysis for Data Collected at the First Breastfeeding Session

\begin{tabular}{|lccc|}
\hline Breastfeeding Variable & R2 & F & p \\
\hline Areola grasp & .199 & 4.288 & .002 \\
Latch & .025 & 0.328 & .858 \\
Letdown & .202 & 3.221 & .020 \\
Longest sucking burst & .133 & 2.010 & .107 \\
Sucking & .169 & 2.600 & .047 \\
Swallowing & .125 & 1.814 & .140 \\
Note. Each breastfeeding variable was used as a dependent variable, \\
and the components of the Neurologic and Adaptive Capacity Score \\
(adaptive behaviors, active tone, passive tone, and primary reflexes) \\
were used as independent variables. Models tested were Breastfeeding \\
variable (i.e., areola grasp, etc.) = Adaptation + Active Tone + Passive \\
Tone + Primary Reflexes.
\end{tabular}

Pearson correlations between the variables were reviewed first to examine the possibility of a linear relationship between the variables. The variables "infant behavioral state," "time infant held," "rooting," and "environmental influences" had no significant correlation with the NACS and were not included in the final model.

Separate regression analysis was performed with each of the breastfeeding behaviors as the dependent variable and the independent variables, which were the neurobehavioral categories on the NACS: adaptive behaviors, active tone, passive tone, and primary reflexes. The model tested by multiple regression was Breastfeeding behavior (each behavior on the PIBBS was tested individually as well as the total score) =Adaptation + Active Tone + Passive Tone + Primary Reflexes (each category of the NACS).

Cord blood drug levels were compared to the epidural infusion time using regression analysis. Regression analysis was then used to determine to what degree the amount of drug present at delivery affected the PIBBS or the N A C S variables.

Sample size was determined for comparative analysis using a medium effect at $80 \%$ power and a .05. Clinically, a medium effect or one half of a standard deviation between the two groups was considered the minimum difference worth detecting. A type I error of $0.05 \%$ was used for all tests of statistical significance. Data were analyzed using the SPSS PC computer program.

\section{Results}

When regression analysis was performed on the individual breastfeeding behaviors and the NACS, $p=.05$ was achieved with breastfeeding behaviors areolar grasp, letdown, and sucking at the initial data collection session
TABLE 4

Regression Analysis for Data Collected at 24 Hrs of Age

\begin{tabular}{|lrrc|}
\hline Breastfeeding Variable & R2 & F & p \\
\hline Areola grasp & .198 & 3.155 & .022 \\
Latch & .197 & 3.136 & .022 \\
Letdown & .196 & 3.115 & .023 \\
Longest sucking burst & .110 & 1.561 & .199 \\
Sucking & .165 & 2.354 & .066 \\
Swallowing & .053 & $\mathbf{0 . 7 0 7}$ & .591 \\
Weight gain & .192 & 3.022 & .026 \\
Note. Each breastfeeding variable was used as a dependent variable, \\
and the components of the Neurologic and Adaptive Capacity Score \\
(adaptive behaviors, active tone, passive tone, and primary reflexes) \\
were used as independent variables. Models tested were Breastfeeding \\
variable (i.e., areola grasp, etc.) = Adaptation + Active Tone + Passive \\
Tone + Primary Reflexes. \\
\end{tabular}

as indicated in Table 3. The higher the score on the NACS, the more likely the newborn was to take the nipple and areola into his mouth, to have repeated long sucking bursts, and to have the mother feel letdown. When regression analysis was performed on breastfeeding behaviors and the NAC S at the second data collection session at 24 hrs postbirth, $p=.05$ was achieved with breastfeeding behaviors areolar grasp, latch, letdown, and weight change as indicated in Table 4. At 24 hrs of age, the higher the score on the NACS, the greater the likelihood the newborn would take the areola into his mouth, remain latched on, the mother feel letdown, and an increase noted in the postfeeding weight of the newborn. The number of feedings between the first and second assessment were also taken into consideration as contributing to the postfeeding weight gain of the newborn but were not found to be factor-affecting results. The number of times mothers in the unmeclicated group fed their newborns ranged from 1 to 7 , with a mean of 4.1 , and the number of times the mothers in the meclicated group fed their newborns ranged from 3 to 8 , with a mean of 5.4 .

Cord blood drug levels for bupivacaine ranged from 0.1 nanograms to 98.6 nanograms/rnl. The level of bupivacaine was related to the length of time the epidural was infusing $\left(\mathrm{R}^{2}=.42, F=17.631, p=.000\right)$. The longer the epidural was in place, the greater the concentration of bupivacaine in the cord blood at delivery. Cord blood drug levels for fentanyl ranged from nondetectable (fentanyl was not detectable below the level of 0.01 nanograms/rnl) to 0.426 nanograms $/ \mathrm{ml}$. The level of fentanyl was not related to the amount of time the epidural was in place $\left(\mathrm{R}^{2}=.04, F=0.956, p=.338\right)$. 


\section{Whe higher the newborn scores in \\ neurobehavioral functioning, the higher the \\ newborn scores on breastfeeding behaviors.}

The level of bupivacaine was significant in relation to passive tone, active tone, and the total score on the N A C S during the initial exam but not during the second exam at 24 hrs of life. The results are presented in Table 5. There were no significant findings between the levels of bupivacaine and any of the breastfeeding variables on the PIBBS. There were no significant findings between fentanyl levels and any of the NACS or breastfeeding behaviors on the PIBBS.

\section{Discussion}

The purpose of this study was to determine whether neurobehavioral responses in the newborn were predictive of the newborn's ability to demonstrate breastfeeding behaviors in the 1st day of life. Newborns who were exposed to a standard epidural infusion of bupivacaine and fentanyl during labor were included in the sample as well as newborns who were not exposed to any medications during labor to see if the narcotic exposure had any central nervous system depressant effect that influenced newborn neurobehavior or breastfeeding behaviors. The supposition tested was that if newborn neurobehavior was affected, then breastfeeding behaviors, which included primary reflexes and adequate tone to maintain latchon, sucking, and swallowing, would also be affected.

This was based on physiologically described mechanisms involved in the breastfeeding process for human infants. Specifically, for a baby to latch on, she must draw the areola and nipple into the mouth and hold it in place. This is primarily done by suction (Ardran, Kemp, \& Lind, 1958). If the infant cannot exert adequate suction, she will not be able to retain the breast in the mouth. Inadequate suction is often described by the mother as feeling the baby is not "attached" or that her breast is just lying in the baby's mouth. The tone of the facial and intraoral muscles and movement of the tongue necessary for this action are dependent on the function of the cranial nerves (Dickason, Silverman, \& Kaplan, 1998). In addition to using her tongue, the infant must also use her lips to create an effective seal around the breast. Without an effective seal, the infant will have difficulty creating the negative pressure within the oral cavity necessary to retain the breast within the mouth (Smith, Erenberg, Nowak, \& Franklin, 1985). Mothers often describe this ineffective
TABLES

$\boldsymbol{R}_{\text {eg }}$ ression Analysis Using Bupivacaine Level in the Cord Blood as the Independent Variable and the Components of the Neurologic and Adaptive Capacity Score

\begin{tabular}{|lccc|}
\hline Breastfeeding Variable & R2 & F & P \\
\hline Active tone & .28 & 9.347 & .005 \\
Adaptive & .04 & 0.099 & .756 \\
Passive tone & .15 & 4.249 & .050 \\
Primary Reflexes & .00 & 0.000 & .984 \\
Note. Adaptive behaviors, active tone, passive tone, and primary \\
reflexes were used separately as dependent variables. Models tested \\
were Active tone = Bupivacaine Level found in the Cord Blood, and so \\
on.
\end{tabular}

seal as feeling that their breast just falls out of the baby's mouth. To maintain a seal, it has been suggested that the infant will require adequate muscle tone to support the weight of the maternal breast on the lower mandible (Shrago \& Bocar, 1990). These actions are highly dependent on the facial and accessory cranial nerves (Dickason et al., 1998 ).

In this study, a relationship between sections of the N A C S and certain breastfeeding behaviors was demonstrated. Upper body tone was tested using the NAC S as well as the newborns' primary reflexes and their behavioral or activity level. These same parameters were evaluated in relation to breastfeeding because for the newborn to feed successfully, the newborn must be in an awake state, possess muscle tone to latch onto the breast and maintain the breast in the mouth, generate enough intraoral pressure to form a maternal teat, and maintain enough tone in the tongue to massage the areolar ducts and rhythmically coordinate the tongue and facial muscles to achieve a suck-swallow-breathe cycle (Klaus, 1987; Lawrence, 1994).

In this study, the newborns' ability to latch onto the breast was significantly related to passive tone, primary reflexes, and adaptive responses. This is not surprising, because adaptive responses test newborns' abilities to respond to their environment. The greater and more sustained the response, the more awake and alert the newborn. Awake, alert newborns tend to feed better. Tests of passive tone such as the scarf sign and recoil of the arms and legs were also related to newborn breastfeeding behaviors. High scores on passive tone were demonstrated in newborns with brisk reproducible responses. These newborns tended to tightly flex and bring their heads forward in good breastfeeding positions. Primary reflexes include assessment of the newborn's ability to flex and extend the head, neck, and extremities. They also provide 
for protective reflexes such as gag, suck, and swallow. The newborn would have difficulty latching onto the breast if he could not achieve flexion. Newborns in extension would have difficulty maneuvering their head forward to reach the breast.

Letdown was significantly related to active tone in this study population. This relationship is difficult to interpret, however, because letdown as defined by the PIBBS refers to the mother's awareness of the sensation rather than the presence of the reflex. It could be postulated that if letdown is occurring, then it is reasonable to expect a change in the newborn's sucking pattern, presence of swallowing, and some weight gain postfeeding. The findings of this study support this because active tone was correlated to the longest sucking burst, swallowing, and weight change postfeeding in the newborn. Active tone includes the active contraction of the ne.ck flexors and extensors. These muscles allow the newborn to maneuver the head and maintain a spatial relationship to the body and other objects. Absence of active tone would not allow the newborn to lift the head or maneuver to find the breast. Once attached to the breast, active tone allows the newborn to maintain attachment by preventing the head from falling forward with gravity and breaking suction, or in the case of hypertonicity, extending the head backward, pulling the breast out of the mouth.

\section{Conclusions}

The results of this study demonstrated a relationship between certain neurobehaviors and breastfeeding behaviors in normal 1-day-old newborns. The lower the score on the NACS, the lower the score on the PIBBS, and the less weight gain in the newborn postfeeding. This indicates the importance of recognizing factors that affect neurobehavioral functioning at birth in relation to the newborn's ability to initiate breastfeeding behaviors. Neurobehavioral assessments at birth may prove clinically helpful as a screening assessment in predicting which newborns may need additional assistance or may need additional time to successfully feed.

\footnotetext{
Careful attention should be given to breastfeeding newborns influenced by any variable that affects, even temporarily, their central nervous system functioning.
}

Exposure to epidural analgesia during labor process was examined as a possible central nervous system depressant variable in this study. Although epidural analgesia predicted lower NACS scores, the exposure to epidural analgesia was not sufficient to affect central nervous system functioning. This study will need to be repeated with newborns experiencing greater exposure to central nervous system depressant events to further determine the exact nature of the relationship. This study did, however, show that even in normal, healthy newborns, slight differences in central nervous system functioning was sufficient to affect breastfeeding in the 1st day of life.

\section{REFERENCES}

Amiel-Tison, C., Barrier, G., \& Shnider, S. (1982). A new neurologic adaptive capacity scoring system for evaluating obstetric medication in full term infants. Anesthesiology, 56, 340-346.

Ardran, G., Kemp, E, \& Lind, J. (1958). A cineradiographic study of breastfeeding. British Journal of Radiology, 31, 156-162.

Dickason, E., Silverman, B., \& Kaplan, J. (1998). Materna/infant nursing care. St. Louis: Mosby.

Hill, P., Hummenick, S., Brennan, M., \& Wooley, D. (1997). Does early supplementation affect long-term breastfeeding? Clinical Pediatrics, 36(6), 345-359.

Hodgkinson, R., Blatt, M., \& Wang, C. (1978). Double-blind comparison of the neurobehavior of neonates following the administration of different doses of meperidine to the mother. Canadian Anesthetists Society Journal, 25(5), 405-411.

Klaus, M. (1987). The frequency of suckling: A neglected but essential ingredient of breastfeeding. Obstetrics and Gynecology Clinics of North America, 4(3), 623-632.

Krishna, V., \& Plichta, S. (1998). The role of social support in breastfeeding promotion: A literature review. Journal of Human Lactation, 14(1), 41-45.

Lawrence, R. (1994). Breastfeeding: A guide for the medical profession. St. Louis: Mosby.

Nygvist, K., Rubertson, C., Ewaksm, U., \& Sjoden, P. (1996). Development of the Preterm Infant Breastfeeding Behavior Scale (PIBBS). A study of nurse-mother agreement. Journal of Human Lactation, 12(3), 207-219.

Radzyminski, S. (2003). The effect of ultra low dose epidural analgesia on newborn breastfeeding behaviors. Journal of Obstetric, Gynecologic, and Neonatal Nursing, 32(3), 322-331.

Righard, L., \& Alade, M. (1990). Effect of delivery room routines on success of first breastfeed. Lancet, 336, 11081107.

Scanlon, J., Brown, W., Weiss, J., \& Alper, M. (1974). Neurobehavioral responses of newborn infants after maternal epidural anesthesia. Anesthesiology, 4(40), 121-128. 
Shrago, L., \& Bocar, D. (1990). The infants contribution to breastfeeding. Journal of Obstetric, Gynecologic, and Neonatal Nursing, 19, 209-215.

Smith, W., Erenberg, A., Nowak, A., \& Franklin, E. (1985). Physiology of sucking in the normal term infant using real-time ultrasound. Radiology, 156, 379-381.
Sharon Radzyminski, PhD,JD, RN, is an assistant professor for nursing and graduate program director in the School of Nursing at Cleveland State University, Cleveland, $\mathrm{OH}$.

Address for correspondence: Sharon $\operatorname{Rad}_{z}$ minski, PhD, JD, RN, School of Nursing, Cleveland State University, 2121 Euclid Avenue RT 928, Cleveland, $\mathrm{OH}$ 44115. E-mail: s.radzminski@csuohio.edu. 